\title{
The role of lipopolysaccharides in diabetic retinopathy
}

\author{
Xinran Qin ${ }^{1}$ and Haidong Zou ${ }^{1,2,3,4,5^{*}}$
}

\begin{abstract}
Diabetes mellitus (DM) is a complex metabolic syndrome characterized by hyperglycemia. Diabetic retinopathy (DR) is the most common complication of DM and the leading cause of blindness in the working-age population of the Western world. Lipopolysaccharides (LPS) is an essential ingredient of the outer membrane of gram-negative bacteria, which induces systemic inflammatory responses and cellular apoptotic changes in the host. High-level serum LPS has been found in diabetic patients at the advanced stages, which is mainly due to gut leakage and dysbiosis. In this light, increasing evidence points to a strong correlation between systemic LPS challenge and the progression of DR. Although the underlying molecular mechanisms have not been fully elucidated yet, LPS-related pathobiological events in the retina may contribute to the exacerbation of vasculopathy and neurodegeneration in DR. In this review, we focus on the involvement of LPS in the progression of DR, with emphasis on the blood-retina barrier dysfunction and dysregulated glial activation. Eventually, we summarize the recent advances in the therapeutic strategies for antagonising LPS activity, which may be introduced to DR treatment with promising clinical value.
\end{abstract}

Keywords: Lipopolysaccharides, Diabetic retinopathy, Gut leakage, Dysbiosis, Inflammation, Blood-retina barrier, Retinal glia

\section{Background}

Diabetes mellitus (DM) is a chronic metabolic syndrome characterized by abnormally high blood glucose levels. Absolute or relative lack of insulin caused by pancreatic $\beta$-cell dysfunction, insulin resistance, or both is the main reason for hyperglycemia [1]. The prevalence of DM was approximately 460 million people worldwide in 2019 , and it is expected to rise to 700 million by 2045 [2], which poses a significant threat to global health. Hyperglycaemia, dyslipidaemia, insulin resistance, and metabolic pathway dysregulation are considered the core pathophysiological mechanisms of DM, leading to a series of complications involving multiple organ functions [1]. With an in-depth understanding of DM, oxidative stress, immune abnormalities, genetics, and

*Correspondence: zouhaidong@sjtu.edu.cn

1 Department of Ophthalmology, Shanghai General Hospital, Shanghai Jiao Tong University School of Medicine, Shanghai, China

Full list of author information is available at the end of the article epigenetics are currently considered significant contributors to the development of DM and its complications [3]. Diabetic retinopathy (DR) is the principal ophthalmic complication of DM, and it is clinically categorised into non-proliferative DR (NPDR) and proliferative DR (PDR) based on ophthalmoscopically visible microangiopathies [4]. Approximately $35 \%$ of diabetic patients have different degrees of retinopathy, and nearly $10 \%$ of them can progress to blindness [5]. In the Western world, DR is the primary cause of blindness in the working-age population.

Lipopolysaccharides (LPS), also known as endotoxins, are a key component of the outer membrane of gram-negative bacteria released after lysis. LPS has an amphiphilic and tripartite structure comprising a highly variable $\mathrm{O}$ antigen, core oligosaccharide, and lipid $\mathrm{A}$ (the main virulence factor) [6]. As a vital pathogenassociated-molecule-pattern (PAMP) in gram-negative bacteria, LPS can induce an innate immune defence and trigger inflammatory cascades in the host [7]. Several original author(s) and the source, provide a link to the Creative Commons licence, and indicate if changes were made. The images or other third party material in this article are included in the article's Creative Commons licence, unless indicated otherwise in a credit line to the material. If material is not included in the article's Creative Commons licence and your intended use is not permitted by statutory regulation or exceeds the permitted use, you will need to obtain permission directly from the copyright holder. To view a copy of this licence, visit http://creativecommons.org/licenses/by/4.0/. The Creative Commons Public Domain Dedication waiver (http://creativeco mmons.org/publicdomain/zero/1.0/) applies to the data made available in this article, unless otherwise stated in a credit line to the data. 
studies have found an association between high-level serum LPS and the progression of diabetic microvascular complications [8], which suggests that LPS may participate in retinal pathology.

This review examines the clinical and preclinical evidence on the association between LPS and DR, explores the possible role of LPS in the progression of DR, and summarizes recent advances in potential strategies for antagonising LPS in DR treatment.

\section{Increased levels of serum LPS in diabetic patients}

Accumulating clinical evidence has demonstrated elevated serum LPS in diabetic patients with or without overt infection, also known as metabolic endotoxemia [8]. Therefore, the source of serum LPS in diabetic patients deserves further examination.

Gut microbiota-derived LPS should be of primary consideration. Gut microbiota is a group of $10^{11}-10^{12}$ bacteria that colonise the human intestine and interact with the host throughout their lifespan. Its existence plays an essential role in maintaining intestinal homeostasis [9]. As a diverse ecosystem subjected to natural variations, the gut microbiota is highly flexible in different individuals and is implicated in numerous diseases, including DM $[10,11]$. Significant shifts in the ratios of dominant phyla or outgrowth of pathobionts (symbiotic gut bacteria that may become pathogenic when appearing in large numbers) can result in a disease-prone status, referred to as dysbiosis $[12,13]$. To date, sufficient studies have revealed marked differences in the gut microbiota composition of diabetic patients. A decline in microbial diversity and the growth of Firmicutes over Bacteroidetes are the principal features of the gut microbiome in diabetic patients [14]. The increased abundance of gram-negative opportunistic pathogens (some strains of Bacteroides, Proteobacteria, Enterobacter, and Escherichia) and decreased abundance of short-chain fatty acid (SCFA)-producing bacteria (such as Fecalibacterium prausnitzii, Eubacterium rectale, and some species in Roseburia and Lachnospira) are also universal traits in diabetic patients and experimental animals [15-17]. The contributing factors of dysbiosis in DM warrant a more detailed discussion. Currently, the mainstream belief attributes dysbiosis to four sources, including nutrients, host immunity, intestinal mucosa, and medication [12]. For example, high-fat and low-fibre diets in type II diabetes are often associated with increased Proteobacteria in the gut, bile acid pool, circulating LPS, and decreased levels of SCFA-producing bacteria [18-20]. The standard anti-hyperglycemic drug, metformin, increases Escherichia coli in the gut [21]. Furthermore, diabetic patients with DR have a significant imbalance in anti-endotoxin immunity during the disease course. The highest concentration of serum anti-LPS-IgA was detected in patients with NPDR that had macular oedema, aneurysms, haemorrhages, and portions of solid exudate; in addition to vascular changes, much lower concentrations of anti-LPS-IgA were identified in PDR, and the lowest concentrations of antiendotoxin antibodies were found in patients with PDR complicated by neovascular glaucoma [22].

Metabolic endotoxemia can be aggravated by gut leakage in DM. Generally, tight junctions between intestinal epithelial cells (IECs) form an effective barrier for the healthy to resist the translocation of gut bacteria and their components into the bloodstream. Nevertheless, the extensively identified gut barrier dysfunction in diabetic patients may provide an opportunity for bacterial products to enter the bloodstream and elicit deleterious systemic effects [23, 24]. Previous studies have widely hypothesized that hyperglycemia is the culprit of gut leakage in diabetic patients through glucose transporter 2-dependent transcriptional reprogramming of IECs [25]. With substantial exploration of dysbiosis, its role in the increased gut permeability is gaining attention. For example, the decrease in Bifidobacterium can lead to a deficiency of glucagon-like peptide-2, which downregulates the expression of tight junction proteins between IECs [26], and the increase in some invasive strains of Escherichia may elicit direct damage to the IECs [27], while the decrease in SCFAs impairs the barrier function by influencing adenosine 5'-monophosphate-activated protein kinase activity and the assembly of tight junctions $[17,28]$. Collectively, gut leakage and dysbiosis in diabetic patients allow an increased number of LPS to enter the bloodstream. Circulating LPS can spontaneously selfaggregate owing to its amphiphilic nature [5]. The aggregates quickly bind to LPS-binding protein (LBP) in the serum, and LBP can catalytically transfer them to soluble cell-differentiation 14 (sCD14) [29, 30]. Unique in their interactions with LPS, LBP and sCD14 can be designated soluble pathogen-recognition receptors and indicators of LPS activity. Ultimately, $90 \%$ of serum LPS can be captured by liver macrophages (i.e., Kupffer cells) within $1 \mathrm{~h}$ and inactivated by acyloxyacyl hydroxylase. However, the impaired Kupffer cell function in diabetic patients dampens the clearance of LPS [31], leading to the persistence of high-level serum LPS. (Fig. 1).

\section{Endotoxemia and the progression of DR are closely related}

For decades, DR has been regarded as a microvascular disease, with advances in diagnostic technology, however, DR manifests early signs of neurofunctional alterations even before the appearance of vascular pathology [32]. Accordingly, the comprehensive definition of DR 

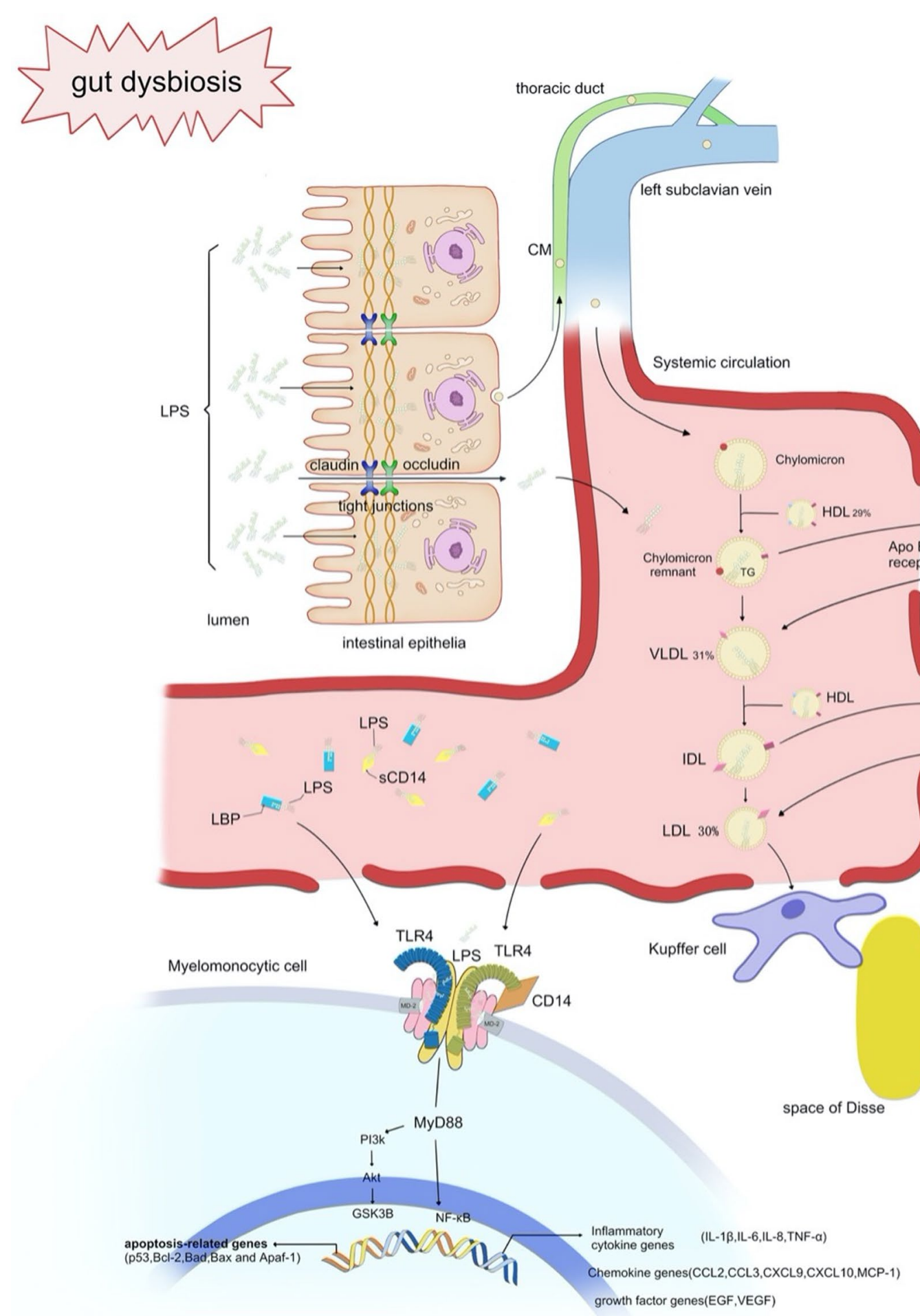

Fig. 1 Schematic illustration of the pathogenesis of endotoxemia in diabetes mellitus, depicting the processes of translocation from the intestinal epithelial cells, transport in the circulation, and subsequent elimination by hepatic Kupffer cells, as well as cellular signaling in response to LPS at a molecular level 
includes retinal vasculopathy and neuropathology, highlighting its complex and dynamic pathogenesis.

Hyperglycemia has long been hypothesized as the central pathophysiology of DR that causes four classical metabolic abnormalities in the retina: the activation of protein kinase $\mathrm{C}$ and the hexosamine pathway, the polyol pathway flux, and the production of advanced glycation end products (AGEs) [33]. The downstream effects mainly include the osmotic damage to retinal capillaries, oxidative stress in multiple retinal cells, and increased expression of vascular endothelial growth factor (VEGF) [34]. Specifically, AGE accumulation has been considered to play an indispensable role in the pathogenesis of DR and correlate with disease severity. At the molecular level, AGEs can damage the function and structure of various proteins by nonenzymatic crosslinks, especially antioxidant enzymes and collagens, which are detrimental to retinal capillary tonicity [35]. In addition, the interaction with its receptor contributes to the erroneous activation of multiple proinflammatory and proangiogenic pathways [36]. Of note, a recent study reported the clinical use of skin autofluorescence to assess AGE accumulation, which can serve as a non-invasive and reliable biomarker for identifying the patients at risk of sightthreatening DR [37].

Growing evidence has revealed the chronic low-grade inflammatory nature of DR. Elevated concentrations of inflammatory cytokines, such as interleukin (IL)-1 $\beta$, IL-6, IL-8, tumour necrosis factor $\alpha$ (TNF- $\alpha)$, chemoattractant protein-1 (MCP-1), and VEGF, have been detected in the ocular tissues (vitreous and/or aqueous humour) of diabetic patients at different stages of DR [38-41]. Additionally, increasing numbers of anti-inflammatory drugs exhibit beneficial outcomes for DR treatment. For example, intravitreal steroids (such as Ozurdex and Iluvein) are approved to handle DR patients with severe complications [42, 43], and clinical trials to determine the efficacy of IL-6 inhibitors, such as EBI-031(clinicaltrials.gov ID: NCT02842541) and tocilizumab (clinicaltrials.gov ID: NCT02511067), have been carried out. Furthermore, approved drugs (such as aflibercept, bevacizumab and ranibizumab) that are extensively used in clinical practice can also work through inflammatory pathways of the retina [44].

The retina has long been regarded as an immuneprivileged organ, as it is physically isolated from peripheral pathogens by the blood-retina barrier (BRB) [45]. BRB is a multicellular structure that can be divided into two layers: The inner layer is composed of tight junctions between the retinal endothelial cells (RECs) resting on the basement membrane, which is covered by astrocytes, Müller cells, and pericytes, that form the retinal neurovascular unit (NVU) [46]. The intactness of the NVU is a prerequisite for vascular integrity and for effectively preventing the passage of macromolecules across the barrier. The outer layer consists of tight junctions between retinal pigment epithelial cells (RPE), which is responsible for the separation of choroidal vasculature from neuroretina and permits the flux of specific molecules into the retina to regulate the dynamic balance of retinal metabolism [47]. Based on the current understanding of the fundamental role of inflammation in the pathogenesis of DR, high serum LPS in DM may be a significant candidate contributor to DR progression. Accumulating evidence suggests that periphery LPS have access to the eye and are sufficient to induce innate immune responses in the retinal cells, with or without BRB dysfunction, and there do exist traces that systemic LPS challenge may be associated with the progression of DR.

First, there may be high concentrations of LPS in the diabetic eyes. A previous study identified significantly increased levels of LBP and sCD14 in the vitreous humour of PDR patients, which indirectly demonstrated an increase in the intraocular LPS in DR [48]. Several studies have also found an increase in the level of sCD14 in the aqueous humour of patients with diabetic macular oedema (DME), a sight-threatening complication of DR. The elevated concentration of SCD14 is associated with that of VEGF, which is crucial for the development of DR. Combined with the analysis of optical coherence tomography images, it was also found that the higher sCD14 levels in diffuse DME patients closely correlated with the increase in the number of optically highly reflective foci in the retina, suggesting increased severity of retinal inflammation [49]. Despite the lack of direct evidence on the existence of LPS in diabetic eyes, a localization study confirmed the ocular distribution of LPS following intravenous injection in rabbits [50]. This may have resulted from the circular openings of approximately $800 \AA$ in diameter on the choroidal capillary wall [51].

Second, peripheral LPS can induce and aggravate the retinal pathology. Repeated intraperitoneal injections of low-dose LPS gave rise to pathological manifestations mimicking DR in healthy mice, including activation, proliferation, and migration of retinal microglia; infiltration of the retina by monocyte-derived macrophages; and the highly reproducible breakdown of the BRB accompanied by subretinal fluid accumulation [52]. In parallel, systemic administration of low-dose LPS caused a 3.5-fold increase in endothelial cell injury and thinning $(10-13 \mu \mathrm{m})$ of the posterior retina in diabetic mice [53], further deterioration of neural function, augmented loss of photoreceptors, worsening of synaptic connectivity, and an increased number of activated microglia in $\mathrm{P} 23 \mathrm{H}$ 
rats (a retinal neurodegenerative animal model) [37-39, 54].

\section{Possible mechanisms of LPS in the pathogenesis of DR}

\section{General pathogenic mechanisms of LPS}

LPS is a classical exogenous pro-inflammatory and proapoptotic mediator that requires $\mathrm{CD} 14$ and Toll-like receptor 4 (TLR4) to elicit cellular responses. Given the lack of transmembrane and intracellular regions, CD14 expressed on the surface of myelomonocytic cells monomerizes LPS aggregates and presents them to the TLR4-myeloid differentiation factor 2 (MD2) complex to transduce signals [55]. In the case of the complex, the $\mathrm{N}$-terminal and central domains of TLR4 provide the charge that is complementary for MD2, subsequently forming a stable heterodimer for specific binding to LPS. With further internalisation, the TLR4-MD2 complex initiates the myeloid differentiation primary response protein 88 (MyD88)-dependent pathway, leading to the activation of multiple transcription factors, especially nuclear factor- $\mathrm{kB}$ (NF- $\mathrm{\kappa B}$ ) and interferon regulatory factor 3 , and the upregulation of the expression of various inflammatory mediators, including pro-inflammatory cytokines (such as IL-1 $\beta$, IL-6, IL-8 and TNF- $\alpha$ ), chemokines $[\mathrm{C}-\mathrm{C}$ motif ligand 2 (CCL2), CCL3, C-X-C motif ligand 3 (CXCL3), CXCL9, CXCL10 and MCP-1], growth factors [epidermal growth factor, platelet-derived growth factor (PDGF), and VEGF], and enzymes for vasoactive substances [inducible nitrous oxide synthase (iNOS) and cyclooxygenase-2 (COX2)]. Besides, AIM2 (absent in melanoma 2)-like receptors and nucleotidebinding oligomerization domain-like receptors (NLRs) are also pivotal receptors of LPS that participate in inflammasome assembly. Inflammasomes interact with the CARD (caspase activation and recruitment domain) of the adaptor protein ASC (apoptosis-related dot-like protein containing CARD) and hydrolyse procaspase-1, thereby recruiting and activating caspase- 1 . Activated caspase- 1 further hydrolyses pro-IL-1 $\beta$ and pro-IL-18 to intensify inflammation [56]. Of note, TLRs and NLRs are widely expressed on retinal cells, and pharmacological blockade of these receptors strongly ameliorates the retinal pathology induced by LPS $[57,58]$.

LPS is also an essential mediator of apoptosis. Recent studies have revealed that intracellular LPS can activate mouse caspase-11 (corresponding to human caspase-4/5) precursors to initiate the process of apoptosis in an atypical manner. Intracellular LPS can spontaneously aggregate into a micelle structure. The negatively charged polar heads of the micelles that locally reach a critical concentration $(10-20 \mu \mathrm{g} / \mathrm{mL})$ can interact with the positively charged residues in caspase-4/5/11, resulting in the oligomerization of the caspase [59]. In addition, the pro-inflammatory cytokines, glutamate, reactive oxygen species (ROS), and nitride oxide (NO) produced by LPS-stimulation can accumulate in the microenvironment of the injured sites, leading to the sequential activation of caspase [60]. Moreover, LPS-TLR4 signalling can upregulate the expression of other pro-apoptotic genes by interacting with the PI3K/AKT/GSK3 $\beta$ and AMPK/ GSK3 $\beta$-Nrf2 pathways [61, 62].

\section{LPS participates in retinal neurodegeneration Microglial activation}

Microglia are the predominant immune cells responsible for surveillance and are the first responders of the innate immune system in the eye. When the surrounding environment is disturbed by pathogenic stimuli, microglia can immediately transform into amoeba-like forms, also called pro-inflammatory phenotypes (M1), induce lowgrade inflammation to defence, and demonstrate strong capacities for proliferation, migration and phagocytosis [63]. With the elimination of the stressors, they restore the transcriptome under surveillance or transform into an anti-inflammatory phenotype (M2), which can release anti-inflammatory cytokines (such as IL-4, IL-10, IL-13, and transforming growth factor) to maintain balance. Nevertheless, the disrupted balance of microglial polarisation has been identified in the retina of diabetic patients at early stages, rendering it a hallmark of DR pathogenesis [64].

Persistent stimulation with LPS may reduce the plasticity of the microglial transcriptome in diabetic eyes, leading to excessive M1 polarisation. This was initially demonstrated by the morphological and phenotypic changes in microglia and a significant increase in inflammatory mediator secretion when activated by LPS [65]. In addition to the canonical LPS-TLR4-MyD88 pathway, LPS may contribute to retinal inflammation by inducing necroptosis in microglia. Necroptosis is a form of cell death that is mediated by receptor-interacting protein kinase $1 / 3$ (RIPK1/3). TLR4 activation is a key initiation step in necroptosis. LPS-activated TLR4 recruits the cytoplasmic adaptor protein TIR-domain containing adaptor inducing interferon- $\beta$ through the endosomal platform. With further interaction between RIPK3 and RIPK1, they act on the effector protein, mixed-lineage kinase like domain, which undergoes a configurational change and translocates to the plasma membrane to increase permeability, leading to necroptosis of microglia with a marked release of inflammatory mediators that, consequently, intensifies LPS-induced retinal inflammation [66].

Microglial activation orchestrates neurodegeneration and vasculopathy in the retina of DR. Activated 
microglia construct an extensively interconnected internet that contributes to cell death in various retinal cells. First, the cytokines released by LPS-activated microglia can trigger the activation of retinal neural glia and elicit neuroinflammatory damage [60]. This cytokine cocktail has been proven neurotoxic, leading to a marked loss of retinal neurons. Moreover, activated microglia with phagocytosed rhodopsin-positive particles were substantially detected in the photoreceptor layer of patients with retinal degenerative diseases [67], suggesting deleterious effects on photoreceptor cell death. Microglial activation also contributes to retinal vascular damage due to its regulatory role in the retinal vasculature. In vitro experiments have shown that activated microglia can promote the angiogenic process and vascular permeability of cocultured RECs through the secretion of VEGF and PDGF [68]. When clearing vascular exudates and cell debris, they also penetrate the basement membrane of the inner $B R B$ to engulf some of the normal RECs leading to an increase in the number of acellular capillaries and resultant vascular leakage. The interaction between pericytes and microglia has been recently uncovered. The upregulation of NADPH oxidase subunits and downregulation of uncoupling protein 2 expressions are found in pericytes co-cultured with LPS-activated microglia, which leads to increased ROS production and secretion of proinflammatory mediators (such as iNOS and TNF $\alpha$ ) in pericytes, negatively influencing BRB maintenance [69].

\section{Reactive gliosis is an indispensable ingredient of neurodegeneration}

Retinal neural glia are neuron-supportive cells with significant functions in immune modulation, metabolism regulation, and nourishment of neurones, which are essential for the homeostasis of the neuroretina network [70]. Specifically, Müller cells are the most widely distributed in the retina, accounting for $90 \%$ of the retinal glia. They form a radial supporting structure along the entire width of the retina, which is critical for metabolism and intercellular communication. Comparatively, astrocytes are confined to the neuroretina, and this distribution is closely related to the presence of retinal vasculature [71]. More importantly, astrocytes provide energy substrates for neurones and secrete neurotrophic factors and antioxidants to promote neural survival [72]. Reactive gliosis refers to the proliferation and activation of Müller cells and astrocytes in response to retinal stress, which includes processes of neuroinflammation, phagocytosis of apoptotic neurones and cell debris, and secretion of neurotrophic factors [73]. The upregulated expression of the glial fibrillary acidic protein (GFAP) is an early sign of reactive gliosis, and increased GFAP expression can be detected in the aqueous humour of NPDR patients, the retina of DR mice, and the LPS-treated glial cells in vitro [74-76], this suggests the pivotal role of gliosis in the progression of DR. Under physiological conditions, a delicate balance is maintained between the damaging and protective effects of reactive gliosis [73]. Long-term exposure to LPS, however, may give rise to chronic gliosis and result in the disturbance of this balance, namely, damage far surpassing protection. Eventually, the inability for activated glia to maintain appropriate support for $\mathrm{BRB}$ and the neuroretina could lead to the exacerbation of neurodegeneration in DR.

In Müller cells, the transcriptomic analysis showed alterations in 78 genes in the 6th month of the course of DR, one-third of these genes was under the regulation of LPS-TLR4 signalling, such as VEGF, intercellular cell adhesion molecule-1 (ICAM-1), IL-1 $\beta$, IL -6, MCP$2, \mathrm{NO}$, and COX2, which are closely related to neuroinflammation [77]. Further, mass spectrometric analysis of Müller cells in response to LPS revealed an increase in the proteins ascribed to antigen-presenting cells (APCs) and function to interact with T-cells [78], suggesting that activated Müller cells may act as noncanonical APCs to participate in the neuroinflammation underlying DR. There is also a program of bidirectional microglia-Müller cell signalling that can mediate adaptive responses within the retina following initial microglial activation [79]. The response of Müller cells may augment initial retinal inflammation and guide the intraretinal mobilisation of microglia through chemokines and cell adhesion. LPS is also a key regulator of hepcidin expression in Müller cells through TLR4-dependent transcriptional reprogramming. The upregulation of hepcidin and subsequent downregulation of ferroportin are associated with increased oxidative stress and apoptosis within the retina in vivo, and chronic exposure to LPS may disrupt iron homeostasis and retinal function [80]. Moreover, LPSactivated Müller cells can exhibit decreased expression and incorrect positioning of potassium channels (Kir4.1), combined with subtle changes in aquaporin, which results in a disturbance of water and potassium transport at the capillary-Müller cell interface and leads to retinal oedema and violation of the NVU function [81]. In astrocytes, LPS can increase the A1-type profile (marked by increased $\mathrm{C} 3$ expression, namely reactive astrocytes) by activating JAK2/STAT3 signalling. A1-type astrocytes can produce multiple inflammatory mediators to aggravate neuroinflammation [82]. It is worth noting that astrocytes require the presence of activated microglia to effectively respond to LPS stimulation in vitro [83], which emphasizes the sequential activation of retinal glia. 


\section{LPS exacerbate retinal vasculature damage and BRB dysfunction}

As the core component of the outer BRB, RPE can transform into a pro-inflammatory state in response to LPS [84], which express high levels of cytokine receptors (including IL-1R,-6R, -8RA, IFNAR1, IFNGR1/2) and secrete a range of inflammatory mediators (including IL-6, -8, -17, -18, IFN- $\gamma$, MCP-1, and VEGF) to induce morphological damage to tight junctions. IL- 6 and IL-8 mediate LPS toxicity through an autocrine feedback loop, which leads to RPE degeneration and further exacerbation of the outer BRB disintegration. As demonstrated in a proteomic analysis of RPE in response to LPS, there is a significant downregulation of proteins related to mitochondrial respiration and cell cycle checkpoints along with an upregulation of proteins related to lipid metabolism, amino acid metabolism, cell-matrix adhesion, and endoplasmic reticulum (ER) stress [85]. The nucleotidebinding domain, leucine-rich repeat-containing family, and pyrin domain-containing 3 (NLRP3) is excessively expressed in RPE treated with LPS in vitro [86]. Furthermore, elevated levels of IL-1 $\beta$, NLRP3, ASC, and caspase-1, along with increased GFAP, Iba-1 (a marker of activated microglia), pro-inflammatory cytokines (TNF- $\alpha$ and IL-6) and pro-angiogenic markers (ICAM-1 and VEGF) were identified in a PDR mouse model, suggesting that the NLRP3 inflammasome plays a pivotal role in the advanced stages of DR. Paradoxically, LPS-treated RPE indicates classical phospholipases D activation that modulates the autophagic process and serves as a protection mechanism [87]. Low-grade activation by systemic LPS administration leads to a preconditioning effect that transiently improves the function and structure of RPE [88]. These protective effects, at least in part, may explain the minor role of endotoxemia in retinal pathology at the very early stage of DM. Long-term exposure to LPS and pro-inflammatory cytokines, however, may induce chronic inflammation that is overwhelmingly detrimental RPE viability, barrier properties and phagocytosis function, thereby contributing to destructive changes in the retinal environment of DR.

Similar inflammatory responses are induced by LPS in the inner BRB. Being extremely susceptible to proinflammatory cytokines, RECs not only become the primary victim of retinal inflammation, but also increase the expressions of chemokines (such as CCL8, CXCL10, and MCP-1) and intracellular adhesion molecules (such as ICAM-1, VCAM-1, and P-selectin), leading to a process referred to as leukostasis [89]. Leukostasis means that peripheral leukocytes begin to marginate, roll, firmly adhere to REC, and migrate into the retina ultimately. This adhesive interaction between leukocytes and RECs is associated with endothelial swelling, an increase in the number of acellular capillaries, and other microangiopathies [90]. Chronic ER stress is another LPS-triggered pathophysiological event in RECs, which results in apoptosis and contributes to retinal degeneration [91]. On the other hand, the damage signals of ongoing retinal inflammation can recruit peripheral macrophages, T-lymphocytes and dendritic cells to migrate into the retina through the leaky blood vessel wall. Subsequently, macrophages that infiltrate the retina can spontaneously undergo transcriptional reprogramming via the TLR4-dependent pathway, expressing the same surface molecules as microglia and performing similar proinflammatory and pro-angiogenic functions to amplify retinal neurovascular injury [92]. The accumulation of immune cells on the surface or within the lumen of retinal vasculature leads to the mechanical occlusion of the retinal vasculature and an increase in non-perfusion areas, which further augments the hypoxic and ischaemic damage to the retina of DR and even induces neovascularization [93, 94] (Fig. 2).

\section{Anti-LPS strategies in DR therapeutics}

The treatment of DR is still challenging, as it involves multiple interplaying pathogenic mechanisms. Photocoagulation laser treatment and intravitreal injection of anti-VEGF agents are significant milestones in the history of DR therapy $[95,96]$. Although both have achieved clinical success through inhibiting neovascularization, there are obvious limitations, such as being effective only for advanced patients with severe neurodegeneration and requiring repeated surgical procedures [97]. Therefore, novel strategies that cover a wide range of mechanisms are urgently needed. Based on the previously discussed role of LPS in the pathogenesis of DR, interventions specifically antagonising LPS may be promising for DR treatment, which warrants further research.

\section{Agents to modulate the gut microbiome and reconstruct barrier integrity}

Probiotics are a class of microorganisms beneficial for regulating the host mucosal immunity, promoting the balance of the gut microbiota, improving the gut barrier function, and maintaining nutrient metabolism [98]. At present, the long-lasting benefits of probiotics on the modulation of the gut microbiota and recovery of gut barrier function in diabetic patients have gained attention [99]. Before formal introduction into clinical practice, large-scale clinical trials are required to determine the long-term efficacy.

In both animal models and clinical trials, orally taking probiotic strains, such as Lactobacillus rhamnosus, Lactobacillus gasseri, Lactobacillus casei, Lactobacillus acidophilus, Streptococcus thermophilus, Lactobacillus 


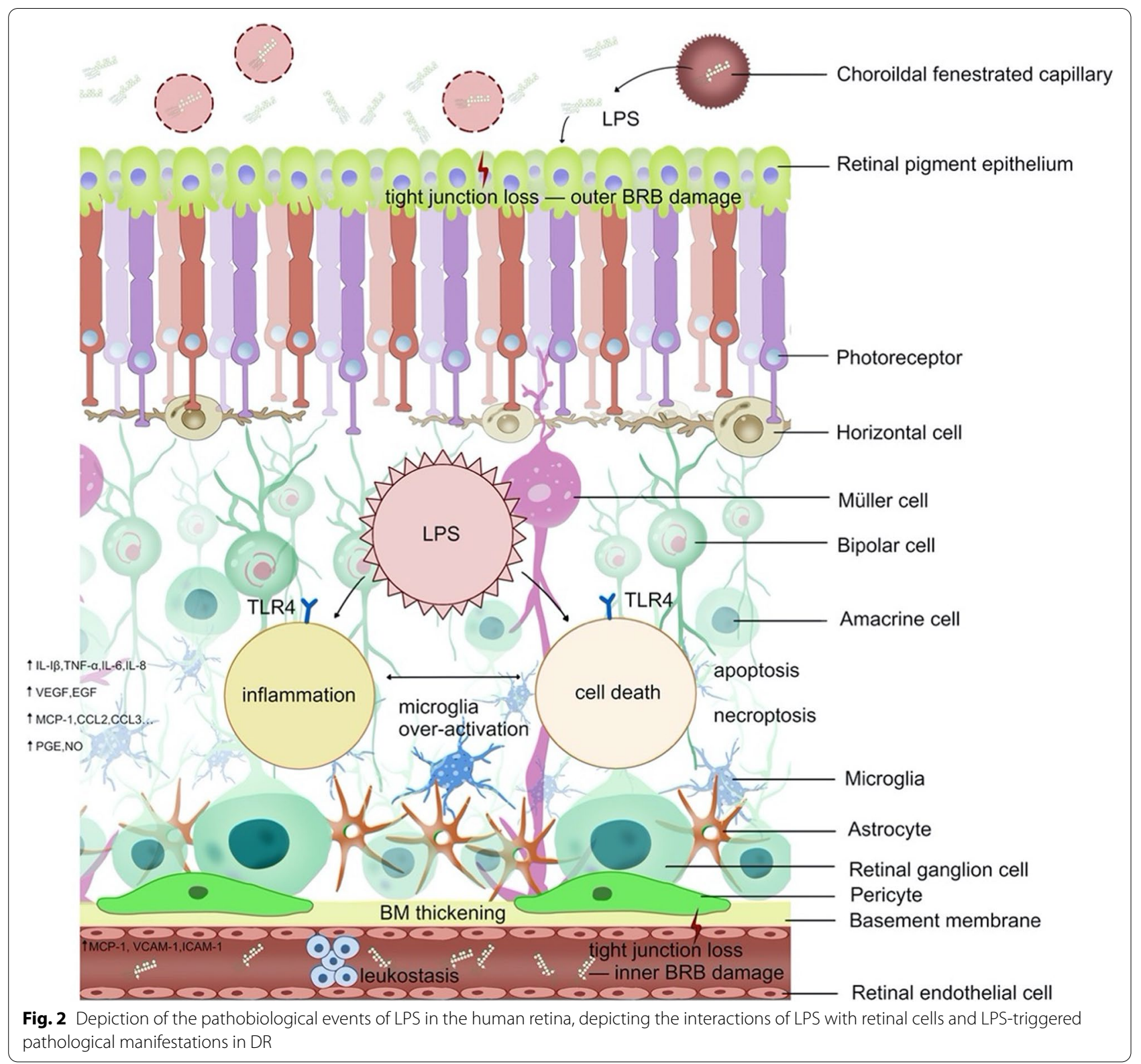

bulgaricus, and Bifidobacterium lactis, for 5-12 weeks exhibited positive effects on DM, including reducing blood glucose, serum LPS, and pro-inflammatory cytokines (TNF- $\alpha$, IL- 6 , and IL- $\beta$ ) levels. Furthermore, a combination of different probiotic strains exhibits even broader benefits on the management of diabetic patients [100-102]. The majority of existing studies merely focused on its value in DM treatment. Yamazaki et al. recent work, however, did suggest that Lactobacillus paracasei KW3110 protects RPE against premature senescence and aberrant expression of tight junction proteins caused by chronic inflammatory stress in vitro and improves the grade of eye fatigue in healthy subjects [103], which strongly suggests the potential of probiotics in chronic eye disorders including DR.

\section{Agents to ameliorate the activity of circulating LPS}

Antimicrobial peptides (AMP), an essential ingredient of innate immunity, are synthesized and secreted by immune cells in response to PAMP signals to eliminate 
pathogens by direct killing effects and immune regulation [104]. AMP can selectively destroy the integrity of bacterial cell membranes, inhibit the synthesis of bioactive macromolecules (such as nucleic acids, proteins, and enzymes), recruit and activate innate immune cells. Defensins are one of the most important groups of AMPs and can be divided into three subfamilies, $\alpha, \beta$, and $\theta$, according to their disulfide bond positions [105]. Compelling evidence has confirmed that human $\beta$ defensin can reduce LPS-induced expression of TNF- $\alpha$ and IL- 6 in both mouse and human macrophages $[106,107]$. Compared to natural AMPs, the newly developed synthetic anti-LPS peptide (SALP) has less cytotoxicity and stronger ability to neutralize LPS toxicity. For example, peptide 19-2.5 can bind firmly to LPS and transform the lipid A into an inactive form, thus impairing LPS activity [108]. Notably, it can also interact with many types of antibiotics and reduce serum TNF- $\alpha$ levels. In an animal septic model, the peptide 19-2.5 successfully lowered the incidence of septic cardiomyopathy and prevented heart failure [109].

Current anti-infection treatment of diabetic patients is facing difficulties. For one thing, the abuse of antibiotics can lead to the emergence of multi-drug resistant bacteria, which poses a devastating threat to handling severe infections at the advanced stages of DM [110]. For another, LPS released by the killed bacteria can be a source of endotoxemia. In contrast, AMP combined with minimal-dose antibiotics can effectively treat the endotoxemia in DM by direct killing and neutralizing LPS toxicity, and help to avoid the antibiotics abuse, which may further become a potential intervention for DR management.

\section{Agents to regulate retinal glial reactivity to LPS}

Glial activation is now considered central to the development of DR [72], so modulating their reactivity to LPS may be helpful to alleviate the LPS-induced retinal inflammation. Olfactory ensheathing cells (OECs), a type of glial cells, can secrete various cytokines involved in immune regulation and neuroinflammation. Xie et al. found that retinal OEC grafts can promote the conversion of microglia from a pro-inflammatory phenotype to an anti-inflammatory phenotype through the JAK-STAT3 pathway, thereby exhibiting anti-inflammatory and neuroprotective capabilities [111]. Jha et al. reported the neuroprotective role of adipose tissue-derived mesenchymal stem cells (ASC-CCMs), a kind of pluripotent stem cells, in the central nervous system inflammatory diseases. Upon LPS stimulation, ASC-CCMs release a variety of bioactive molecules, such as extracellular superoxide dismutase, immunomodulatory proteins (such as indoleamine 2,3-dioxygenase), and TNF-stimulated gene
6 protein (TSG-6). Among them, TSG- 6 can increase the expression of anti-inflammatory mediators in a STAT3dependent manner, thereby promoting the phenotypic transformation of retinal microglia [112]. Besides, Xian et al. have shown the ability of mesenchymal stem cellderived exosomes (MSC-Exos) to combat LPS-induced astrocyte reactivity via the Nrf2-NF- $\mathrm{kB}$ signalling pathway in vivo [113]. Due to the merit of crossing the BRB, exosome has been currently regarded as an efficient drug delivery tool for use in retinopathy. Upon MSC-Exos treatment in this study, the LPS-upregulated expression of pro-inflammatory cytokines can return to normal. Related calcium signal abnormalities and mitochondrial dysfunction were ameliorated, which closely correlate with retinal neurodegeneration. Accordingly, these cell therapies may be beneficial in ameliorating retinal inflammation with promising neuroprotective function, however, clinical trials are still required to determine the clinical value.

Ultimately, microRNA (miRNA), a member of noncoding RNA, has emerged as a pivotal regulator in microglial activation. MicroRNA can cause mRNA silencing or degradation by binding to mRNA, thereby downregulating the expression of its target genes [114]. Currently, increasing numbers of studies have focused on the regulatory role of miRNA in retinal chronic inflammatory conditions. First, a set of dysregulated miRNAs (miR-20a-5p, miR-20a-3p, miR-20b, miR-106a-5p, miR27a-5p, miR-27b-3p, miR-206-3p, and miR-381-3p) are identified in serum and retinas of diabetic mice [115]. They can modify the expression of VEGF, brain-derived neurotrophic factor, and cAMP response element-binding protein 1 , before the occurrence of vasculopathy. In addition, miR-21, miR-223, miR-204, miR-30a, miR-34a, and miR93 also contribute to downregulating microglia activation in vivo or in vitro, and delivery of those miRNAs exhibits neuroprotective effects in the degenerative or ageing retina [116-120]. On the contrary, miR-155 and miR-146a are reported to upregulate inflammatory and apoptotic pathways in microglia, thereby targeting these miRNAs may ameliorate inflammatory response in the degenerative retina [121-123]. With the assistance of nanotechnology and adeno-associated viral vector-based strategy, RNA technology has become an attainable therapeutic approach that can improve retinal drug availability. For example, Amadio et al. reported the use of nanocarriers complexed with small interfering RNA silencing Human antigen $\mathrm{R}(\mathrm{HuR})$ in DR rats. This treatment demonstrated potent retinal protection by significantly dampening the expression of retinal $\mathrm{HuR}$ and its target VEGF [124]. Collectively, regulating the miRNA network may contribute to restraining the retinal 


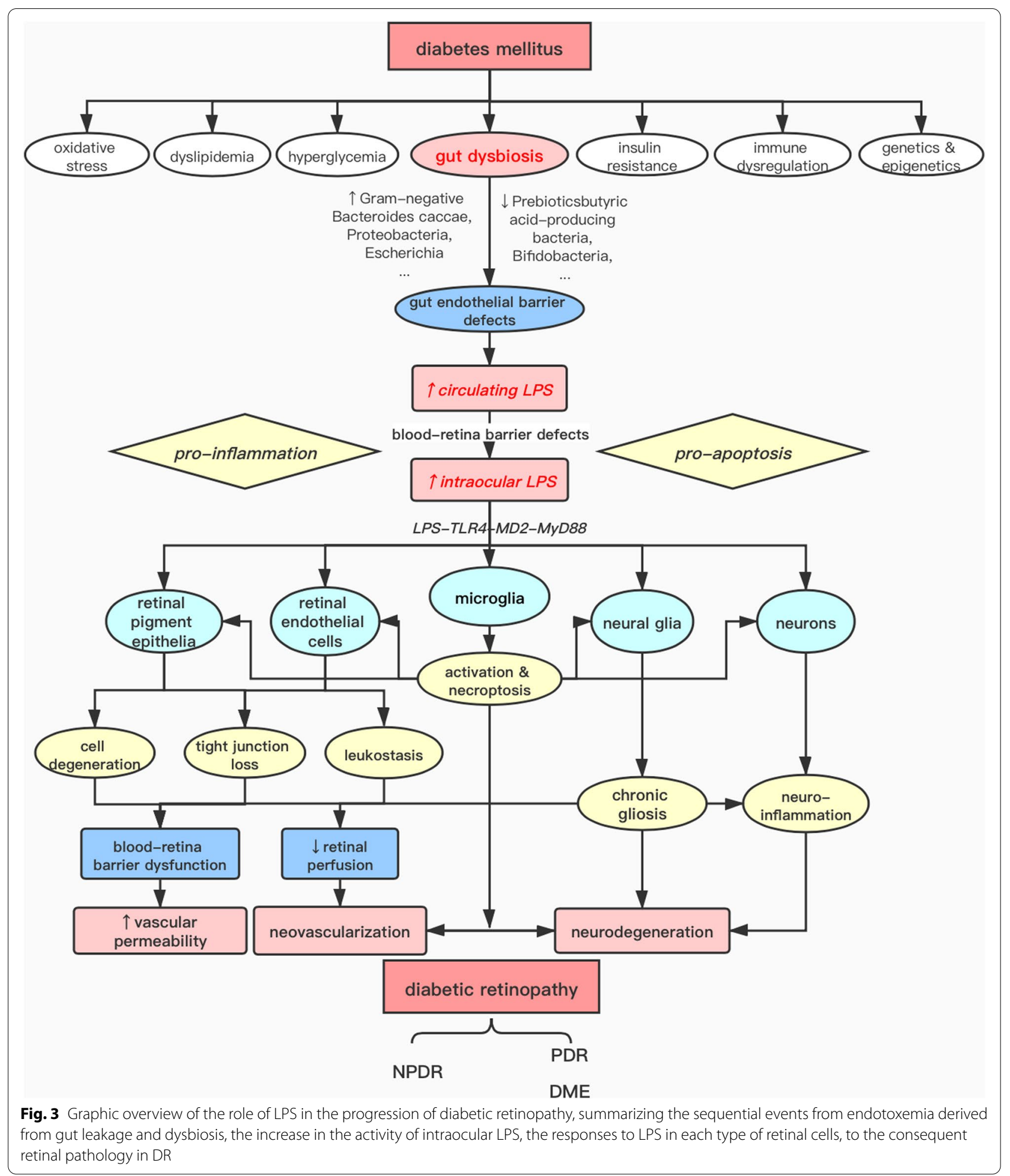

inflammation induced by LPS in DR patients, leading to better neuroprotection in DR management. (Fig. 3).

\section{Conclusions}

This review summarizes the clinical and preclinical research on the association between LPS and DR, 
suggesting a significant role of LPS in the progression of DR. Sufficient studies have found that endotoxemia is common in diabetic patients $[7,8]$, and that gut dysbiosis and defective epithelial barrier may be the culprits. Peripheral LPS have access to the eye through the openings of choroid blood vessels [50], allowing endotoxemia to involve in the pathogenesis of DR. However, this effect is restrained by relatively low LPS activity and intact host immunity at the very early stages of DM. In contrast, there is persistently high-level serum LPS combined with impaired immunity at the advanced stages [22], thus circulating LPS can play a significant part in the progression of DR. By disturbing ocular homeostasis, intruding LPS activate a wide variety of retinal cells to intensify the retinal inflammatory cascades as well as cell degeneration $[57,64,80-88]$, which manifests as aggravation of BRB dysfunction, reduction of retinal blood perfusion, and deterioration of neural dysfunction.

There are great limitations in the current treatment of DR [97]. Given the complexity in retinal structure, multiple pathogenic mechanisms interplay in DR, and nearly every cell component could contribute to the development of DR. Therefore, a single target often has compromised benefits. As the pivotal role of endotoxemia in the pathogenesis of DR has been gradually elucidated, LPS may become a potential target for DR therapeutics. The strategies include reconstruction of the gut microbiota through probiotics, neutralization of the activity of serum LPS by AMPs/SALPs, and regulation of the retinal glial responsiveness to LPS, which are worthy of further research. Overall, the association between LPS and DR reveals the existence of the gut-retina axis, and an indepth understanding of this axis will not only help to elucidate the more comprehensive pathogenic mechanisms of DR and other retinopathies such as age-related macular degeneration and retinopathy of prematurity, but it also sheds lights on better therapeutics.

\begin{abstract}
Abbreviations
AGE: Advanced glycation end product; APC: Antigen-presenting cell; BRB: Blood-retina barrier; CXCL: C-X-C motif ligand; DM: Diabetes mellitus; DME: Diabetic macular oedema; DR: Diabetic retinopathy; ER: Endoplasmic reticulum; GFAP: Glial fibrillary acidic protein; ICAM: Intercellular cell adhesion molecule; IECs: Intestinal epithelial cells; IL: Interleukin; LBP: Lipopolysaccharide-binding protein; LPS: Lipopolysaccharides; MCP: Chemoattractant protein; MD: Myeloid differentiation factor; NLR: Nucleotide-binding oligomerization domain-like receptor; NVU: Neurovascular unit; NPDR: Non-proliferative diabetic retinopathy; PAMP: Pathogen-associated-molecule-pattern; PDR: Proliferative diabetic retinopathy; REC: Retinal endothelial cell; RIPK: Receptorinteracting protein kinase; RPE: Retinal pigment epithelium; sCD: Soluble cell-differentiation 14; TLR4: Toll-like receptor; TNF-a: Tumour necrosis factor a; VEGF: Vascular endothelial growth factor.
\end{abstract}

\section{Acknowledgements}

We thank members of the Zou lab for their support and valuable guidance. All figures were illustrated with SAI.

\section{Authors' contributions}

X.Q. wrote the initial draft, H.Z. revised the manuscript and obtained funding. Both authors read and approved the final manuscript.

\section{Funding}

1. Chinese National Nature Science Foundation (Project number 82071012),

2. The Project of Shanghai Shen Kang Hospital Development Centre (Grant No. SHDC2020CR30538, SHDC2018110).

3. Shanghai engineering research center of precise diagnosis and treatment of eye diseases, Shanghai, China (Project No. 19DZ2250100).

4. The Science and Technology Commission of Shanghai Municipality (Project No. 20DZ1100200).

5. Shanghai public health system three-year plan-Key Subjects (Project No. GWV10.1-XK7).

6. Shanghai General Hospital, Clinical Research CTCCR-2018Z01.

\section{Availability of data and materials}

Not applicable

\section{Declarations}

Ethics approval and consent to participate

Not applicable

Consent for publication

Not applicable

\section{Competing interests}

The authors declare no competing financial or non-financial interests.

\section{Author details \\ ${ }^{1}$ Department of Ophthalmology, Shanghai General Hospital, Shanghai Jiao Tong University School of Medicine, Shanghai, China. ${ }^{2}$ Shanghai Eye Diseases Prevention \& Treatment Center, Shanghai Eye Hospital, Shanghai, China. ${ }^{3}$ Shanghai Engineering Center for Precise Diagnosis and Treatment of Eye Diseases, Shanghai, China. ${ }^{4}$ National Clinical Research Center for Eye Diseases, Shanghai, China. ${ }^{5}$ Shanghai Key Laboratory of Fundus Diseases, Shanghai, China.}

Received: 25 May 2021 Accepted: 3 February 2022

Published online: 22 February 2022

\section{References}

1. American Diabetes Association. Standards of medical care in diabetes — 2016. Diabetes Care. 2016;39:S1-108.

2. Cole JB, Florez JC. Genetics of diabetes mellitus and diabetes complications. Nat Rev Nephrol. 2020;16(7):377-90.

3. Skyler JS, Bakris GL, Bonifacio E, et al. Differentiation of diabetes by pathophysiology, natural history, and prognosis. Diabetes. 2017;66(2):241-55.

4. Grading diabetic retinopathy from stereoscopic color fundus photographs--an extension of the modified Airlie House classification. ETDRS report number 10. Early Treatment Diabetic Retinopathy Study Research Group. Ophthalmology. 1991;98(5 Suppl):786-806.

5. Sabanayagam C, Banu R, Chee ML, et al. Incidence and progression of diabetic retinopathy: a systematic review. Lancet Diabetes Endocrinol. 2019;7(2):140-9.

6. Li H, Liao T, Debowski AW, Tang H, et al. Lipopolysaccharide structure and biosynthesis in helicobacter pylori. Helicobacter. 2016;21 (6):445-61

7. Wu Y, Sun Y, Dong $X$, et al. The Synergism of PGN, LTA and LPS in inducing transcriptome changes, inflammatory responses and a decrease in lactation as well as the associated epigenetic mechanisms in bovine mammary epithelial cells. Toxins (Basel). 2020;12(6):387.

8. Gomes JMG, Costa JA, Alfenas RCG. Metabolic endotoxemia and diabetes mellitus: a systematic review. Metabolism. 2017;68:133-44.

9. Adak A, Khan MR. An insight into gut microbiota and its functionalities. Cell Mol Life Sci. 2019;76(3):473-93. 
10. Gurung $M, L i Z$, You $H$, et al. Role of gut microbiota in type 2 diabetes pathophysiology. EBioMedicine. 2020;51:102590.

11. Tanase D, Gosav E, Neculae E, Costea C, Ciocoiu M, et al. Role of Gut Microbiota on Onset and Progression of Microvascular Complications of Type 2 Diabetes (T2DM). Nutrients. 2020;12(12):3719.

12. Weiss GA, Hennet T. Mechanisms and consequences of intestinal dysbiosis. Cell Mol Life Sci. 2017;74(16):2959-77.

13. Chow J, Tang H, Mazmanian SK. Pathobionts of the gastrointestinal microbiota and inflammatory disease. Curr Opin Immunol. 2011;23(4):473-80.

14. Le Chatelier E, Nielsen T, Qin J, et al. Richness of human gut microbiome correlates with metabolic markers. Nature. 2013;500(7464):541-6.

15. Das T, Jayasudha R, Chakravarthy $S$, et al. Alterations in the gut bacterial microbiome in people with type 2 diabetes mellitus and diabetic retinopathy. Sci Rep. 2021;11(1):2738.

16. Qin J, Li Y, Cai Z, et al. A metagenome-wide association study of gut microbiota in type 2 diabetes. Nature. 2012;490:55-60.

17. Noureldein MH, Bitar S, Youssef N, Azar S, Eid AA. Butyrate modulates diabetes-linked gut dysbiosis: epigenetic and mechanistic modifications. J Mol Endocrinol. 2020;64(1):29-42.

18. Makki K, Deehan EC, Walter J, Bäckhed F. The impact of dietary fiber on gut microbiota in host health and disease. Cell Host Microbe. 2018;23(6):705-15.

19. Fiorucci S, Distrutti E. Bile acid-activated receptors, intestinal microbiota, and the treatment of metabolic disorders. Trends Mol Med. 2015;21(11):702-14

20. Cani PD, Amar J, Iglesias MA, et al. Metabolic endotoxemia initiates obesity and insulin resistance. Diabetes. 2007;56(7):1761-72.

21. Forslund K, Hildebrand F, Nielsen T, et al. Disentangling type 2 diabetes and metformin treatment signatures in the human gut microbiota. Nature. 2015:528(7581):262-6.

22. Zhaboedov GD, Kopaenko Al. Impaired antiendotoxin immunity in patients with diabetic retinopathy and type 2 diabetes mellitus. Vestn Oftalmol. 2005;121(6):29-31

23. Pasini $E$, Corsetti $G$, Assanelli $D$, et al. Effects of chronic exercise on gut microbiota and intestinal barrier in human with type 2 diabetes Minerva Med. 2019;110(1):3-11.

24. Duan $Y$, Prasad R, Feng D, et al. Bone marrow-derived cells restore functional integrity of the gut epithelial and vascular barriers in a model of diabetes and ACE2 Deficiency. Circ Res. 2019;125(11):969-88.

25. Thaiss CA, Levy M, Grosheva I, et al. Hyperglycemia drives intestinal barrier dysfunction and risk for enteric infection. Science. 2018;359(6382):1376-83.

26. Salgaço MK, Oliveira LGS, Costa GN, Bianchi F, Sivieri K. Relationship between gut microbiota, probiotics, and type 2 diabetes mellitus. Appl Microbiol Biotechnol. 2019;103(23-24):9229-38.

27. Zareie M, Riff J, Donato K, et al. Novel effects of the prototype translocating Escherichia coli, strain C25 on intestinal epithelial structure and barrier function. Cell Microbiol. 2005;7:1782-97.

28. Peng L, Li Z-R, Green RS, et al. Butyrate enhances the intestinal barrier by facilitating tight junction assembly via activation of AMP-activated protein kinase in Caco-2 cell monolayers. J Nutr. 2009;139:1619-25.

29. Tsukamoto H, Takeuchi S, Kubota K, et al. Lipopolysaccharide (LPS)binding protein stimulates CD14-dependent Toll-like receptor 4 internalization and LPS-induced TBK1-IKKE-IRF3 axis activation. J Biol Chem. 2018;293(26):10186-201

30. Ryu JK, Kim SJ, Rah SH, et al. Reconstruction of LPS transfer cascade reveals structural determinants within LBP, CD14, and TLR4-MD2 for efficient LPS recognition and transfer. Immunity. 2017;46(1):38-50.

31. Frazier TH, DiBaise JK, McClain CJ. Gut microbiota, intestinal permeability, obesity-induced inflammation, and liver injury. JPEN J Parenter Enteral Nutr. 2011:35(5 Suppl):14S-20S.

32. Tyrberg M, Lindblad U, Melander A, Lövestam-Adrian M, Ponjavic $V$, Andréasson S. Electrophysiological studies in newly onset type 2 diabetes without visible vascular retinopathy. Doc Ophthalmol. 2011;123(3):193-8.

33. Hammes HP. Diabetic retinopathy: hyperglycaemia, oxidative stress and beyond. Diabetologia. 2018;61(1):29-38.

34. Safi SZ, Qvist R, Kumar S, Batumalaie K, Ismail IS. Molecular mechanisms of diabetic retinopathy, general preventive strategies, and novel therapeutic targets. Biomed Res Int. 2014;2014:801269.
35. Stitt AW. Advanced glycation: an important pathological event in diabetic and age related ocular disease. Br J Ophthalmol. 2001;85(6):746-53.

36. Bierhaus A, Hofmann MA, Ziegler R, Nawroth PP. AGEs and their interaction with AGE-receptors in vascular disease and diabetes mellitus. I. The AGE concept. Cardiovasc Res. 1998;37(3):586-600.

37. Takayanagi Y, Yamanaka M, Fujihara J, et al. Evaluation of relevance between advanced glycation end products and diabetic retinopathy stages using skin autofluorescence. Antioxidants (Basel). 2020;9(11):1100.

38. Wu H, Hwang DK, Song X, Tao Y. Association between aqueous cytokines and diabetic retinopathy stage. J Ophthalmol. 2017:2017:9402198.

39. Funatsu H, Yamashita H, Sakata K, Noma H, Mimura T, Suzuki M, Eguchi S, Hori S. Vitreous levels of vascular endothelial growth factor and intercellular adhesion molecule 1 are related to diabetic macular edema. Ophthalmology. 2005;112:806-16.

40. Mao C, Yan H. Roles of elevated intravitreal IL-1 beta and IL-10 levels in proliferative diabetic retinopathy. Indian J Ophthalmol. 2014;62:699-701.

41. Kim M, Kim Y, Lee SJ. Comparison of aqueous concentrations of angiogenic and inflammatory cytokines based on optical coherence tomography patterns of diabetic macular edema. Indian J Ophthalmol. 2015;63:312-7.

42. Campochiaro PA, Brown DM, Pearson A, et al. Long-term benefit of sustained-delivery fluocinolone acetonide vitreous inserts for diabetic macular edema. Ophthalmology. 2011;118:626-635.e2.

43. Pacella F, Agostinelli E, Carlesimo SC, Nebbioso M, Secondi R, Forastiere M, Pacella E. Management of anterior chamber dislocation of a dexamethasone intravitreal implant: a case report. J Med Case Rep. 2016:10:282.

44. Giurdanella G, Anfuso CD, Olivieri M, et al. Aflibercept, bevacizumab and ranibizumab prevent glucose-induced damage in human retinal pericytes in vitro, through a PLA2/COX-2/VEGF-A pathway. Biochem Pharmacol. 2015;96(3):278-87.

45. Chen M, Luo C, Zhao J, Devarajan G, Xu H. Immune regulation in the aging retina. Prog Retin Eye Res. 2019;69:159-72.

46. Antonetti DA, Klein R, Gardner TW. Diabetic retinopathy. N Engl J Med. 2012;366(13):1227-39.

47. Rudraraju M, Narayanan SP, Somanath PR. Regulation of bloodretinal barrier cell-junctions in diabetic retinopathy. Pharmacol Res. 2020:161:105115.

48. Hernández C, Ortega F, García-Ramírez M, Villarroel M, Casado J, et al. Lipopolysaccharide-binding protein and soluble CD14 in the vitreous fluid of patients with proliferative diabetic retinopathy. Retina (Philadelphia, Pa). 2010;30:345-52.

49. Lee $\mathrm{H}$, Jang $\mathrm{H}$, Choi YA, Kim HC, Chung H. Association between soluble CD14 in the aqueous humor and hyperreflective foci on optical coherence tomography in patients with diabetic macular edema. Invest Ophthalmol Vis Sci. 2018;59(2):715-21.

50. Howes EL Jr, Hoffman MA, Ulevitch RJ, Mathison JC, Morrison DC. Ocular localization of circulating bacterial lipopolysaccharide. Exp Eye Res. 1984;38(4):379-89.

51. Bill A, Törnquist P, Alm A. Permeability of the intraocular blood vessels. Trans Ophthalmol Soc U K. 1980;100(3):332-6.

52. Kokona D, Ebneter A, Escher P, Zinkernagel MS. Colony-stimulating factor 1 receptor inhibition prevents disruption of the bloodretina barrier during chronic inflammation. J Neuroinflammation. 2018;15(1):340

53. Vagaja NN, Binz N, McLenachan S, Rakoczy EP, McMenamin PG. Influence of endotoxin-mediated retinal inflammation on phenotype of diabetic retinopathy in Ins2 Akita mice. Br J Ophthalmol. 2013;97(10):1343-50.

54. Noailles A, Maneu V, Campello L, et al. Systemic inflammation induced by lipopolysaccharide aggravates inherited retinal dystrophy. Cell Death Dis. 2018;9(3):350.

55. Ciesielska A, Matyjek M, Kwiatkowska K. TLR4 and CD14 trafficking and its influence on LPS-induced pro-inflammatory signaling. Cell Mol Life Sci. 2021;78(4):1233-61. 
56. Eldridge MJ, Shenoy AR. Antimicrobial inflammasomes: unified signalling against diverse bacterial pathogens. Curr Opin Microbiol. 2015;23:32-41.

57. Ghosh F, Abdshill H, Arnér K, Voss U, Taylor L. Retinal neuroinflammatory induced neuronal degeneration - Role of toll-like receptor-4 and relationship with gliosis. Exp Eye Res. 2018;169:99-110.

58. Hikage F, Lennikov A, Mukwaya A, et al. NF-kB activation in retinal microglia is involved in the inflammatory and neovascularization signaling in laser-induced choroidal neovascularization in mice. Exp Cell Res. 2021;403(1):112581.

59. YiYS. Caspase-11 non-canonical inflammasome: a critical sensor of intracellular lipopolysaccharide in macrophage-mediated inflammatory responses. Immunology. 2017;152(2):207-17.

60. Yang LP, Sun HL, Wu LM, et al. Baicalein reduces inflammatory process in a rodent model of diabetic retinopathy. Invest Ophthalmol Vis Sci. 2009;50(5):2319-27

61. Chen X, Yan X, Guo L. Inhibitory effect of Patrinia on BRL-3A cell apoptosis through the TLR4/PI3K/AKT/GSK3 $\beta$ and TLR4/P38/JNK signaling pathways. Mol Med Rep. 2018;17(4):5344-9.

62. Lv H, Liu Q, Wen Z, Feng H, Deng X, Ci X. Xanthohumol ameliorates lipopolysaccharide (LPS)-induced acute lung injury via induction of AMPK/GSK3ß-Nrf2 signal axis. Redox Biol. 2017;12:311-24.

63. Altmann C, Schmidt MHH. The role of microglia in diabetic retinopathy: inflammation, microvasculature defects and neurodegeneration. Int J Mol Sci. 2018;19(1):110.

64. Bell OH, Copland DA, Ward A, et al. Single Eye mRNA-Seq reveals normalisation of the retinal microglial transcriptome following acute inflammation. Front Immunol. 2020;10:3033.

65. Orihuela R, McPherson CA, Harry GJ. Microglial M1/M2 polarization and metabolic states. Br J Pharmacol. 2016;173(4):649-65.

66. Huang Z, Zhou T, Sun X, et al. Necroptosis in microglia contributes to neuroinflammation and retinal degeneration through TLR4 activation. Cell Death Differ. 2018;25(1):180-9.

67. Scholz R, Sobotka M, Caramoy A, et al. Minocycline counter-regulates pro-inflammatory microglia responses in the retina and protects from degeneration. J Neuroinflammation. 2015;12:209.

68. Ding X, Gu R, Zhang M, et al. Microglia enhanced the angiogenesis, migration and proliferation of co-cultured RMECs. BMC Ophthalmol. 2018;18(1):249.

69. Ding X, Zhang M, Gu R, et al. Activated microglia induce the production of reactive oxygen species and promote apoptosis of co-cultured retinal microvascular pericytes. Graefes Arch Clin Exp Ophthalmol. 2017;255(4):777-88.

70. Bringmann A, Pannicke T, Grosche J, et al. Müller cells in the healthy and diseased retina. Prog Retin Eye Res. 2006;25(4):397-424.

71. Rungger-Brändle E, Messerli JM, Niemeyer G, Eppenberger HM. Confocal microscopy and computer-assisted image reconstruction of astrocytes in the mammalian retina. Eur J Neurosci. 1993;5(8):1093-106.

72. Sorrentino FS, Allkabes M, Salsini G, Bonifazzi C, Perri P. The importance of glial cells in the homeostasis of the retinal microenvironment and their pivotal role in the course of diabetic retinopathy. Life Sci. 2016;162:54-9.

73. Bringmann A, landiev I, Pannicke T, et al. Cellular signaling and factors involved in Müller cell gliosis: neuroprotective and detrimental effects. Prog Retin Eye Res. 2009;28(6):423-51.

74. Vujosevic S, Micera A, Bini S, et al. Aqueous humor biomarkers of muller cell activation in diabetic eyes. Investig Ophthalmol Vis Sci. 2015:56:3913-8

75. Van Hove I, De Groef L, Boeckx B, et al. Single-cell transcriptome analysis of the Akimba mouse retina reveals cell-type-specific insights into the pathobiology of diabetic retinopathy. Diabetologia. 2020;63(10):2235-48.

76. Jang S, Lee JH, Choi KR, Kim D, Yoo HS, Oh S. Cytochemical alterations in the rat retina by LPS administration. Neurochem Res. 2007;32(1):1-10.

77. Gerhardinger C, Costa MB, Coulombe MC, et al. Expression of acutephase response proteins in retinal muller cells in diabetes. Investig Ophthalmol Vis Sci. 2005;46:349-57.

78. Lorenz L, Hirmer S, Schmalen A, et al. Cell surface profiling of retinal müller glial cells reveals association to immune pathways after LPS stimulation. Cells. 2021;10(3):711.
79. Wang M, Ma W, Zhao L, et al. Adaptive Müller cell responses to microglial activation mediate neuroprotection and coordinate inflammation in the retina. J Neuroinflammation. 2011:8:173.

80. Gnana-Prakasam JP, Martin PM, et al. Hepcidin expression in mouse retina and its regulation via lipopolysaccharide/Toll-like receptor-4 pathway independent of Hfe. Biochem J. 2008;411(1):79-88.

81. Liu XQ, Kobayashi H, Jin ZB, Wada A, Nao-I N. Differential expression of Kir4.1 and aquaporin 4 in the retina from endotoxin-induced uveitis rat. Mol Vis. 2007;13:309-17.

82. Zhang HY, Wang Y, He Y, et al. A1 astrocytes contribute to murine depression-like behavior and cognitive dysfunction, which can be alleviated by IL-10 or fluorocitrate treatment. J Neuroinflammation. 2020;17(1):200.

83. Liddelow $S A$, et al. Neurotoxic reactive astrocytes are induced by activated microglia. Nature. 2017;541(7638):481-7.

84. Leung KW, Barnstable CJ, Tombran-Tink J. Bacterial endotoxin activates retinal pigment epithelial cells and induces their degeneration through IL-6 and IL-8 autocrine signaling. Mol Immunol. 2009;46(7):1374-86.

85. Song J, Han D, Lee H, et al. A comprehensive proteomic and phosphoproteomic analysis of retinal pigment epithelium reveals multiple pathway alterations in response to the inflammatory stimuli. Int J Mol Sci. 2020;21(9):3037.

86. Chaurasia SS, Lim RR, Parikh BH, et al. The NLRP3 inflammasome may contribute to pathologic neovascularization in the advanced stages of diabetic retinopathy. Sci Rep. 2018;8(1):2847

87. Bermúdez V, Tenconi PE, Giusto NM, et al. Lipopolysaccharide-Induced Autophagy Mediates Retinal Pigment Epithelium Cells Survival. Modulation by the Phospholipase D Pathway. Front Cell Neurosci. 2019;13:154.

88. Young BM, Ildefonso CJ. Systematic injection of low-dose LPS transiently improves the retina function and structure of a mouse model of geographic atrophy. Adv Exp Med Biol. 2019;1185:57-62.

89. Herdade AS, Silva IM, Calado Â, et al. Effects of diabetes on microcirculation and leukostasis in retinal and non-ocular tissues: implications for diabetic retinopathy. Biomolecules. 2020;10(11):1583.

90. Chen W, Esselman WJ, Jump DB, Busik JV. Anti-inflammatory effect of docosahexaenoic acid on cytokine-induced adhesion molecule expression in human retinal vascular endothelial cells. Invest Ophthalmol Vis Sci. 2005;46(11):4342-7.

91. Huang $H$, Jing $G$, Wang JJ, et al. ATF4 is a novel regulator of MCP-1 in microvascular endothelial cells. J Inflamm (Lond). 2015;12:31.

92. Fouda AY, Xu Z, Shosha E, et al. Arginase 1 promotes retinal neurovascular protection from ischemia through suppression of macrophage inflammatory responses. Cell Death Dis. 2018;9(10):1001.

93. Durham JT, Herman IM. Microvascular modifications in diabetic retinopathy. Curr Diab Rep. 2011;11(4):253-64.

94. Miyamoto K, Khosrof S, Bursell SE, et al. Prevention of leukostasis and vascular leakage in streptozotocin-induced diabetic retinopathy via intercellular adhesion molecule-1 inhibition. Proc Natl Acad Sci U S A. 1999;96(19):10836-41.

95. Singh RP, Elman MJ, Singh SK, Fung AE, Stoilov I. Advances in the treatment of diabetic retinopathy. J Diabetes Complications. 2019:33(12):107417.

96. Aiello LP, Edwards AR, Beck RW, et al. Factors associated with improvement and worsening of visual acuity 2 years after focal/ grid photocoagulation for diabetic macular edema. Ophthalmology. 2010;117(5):946-53.

97. Stitt AW, Curtis TM, Chen M, et al. The progress in understanding and treatment of diabetic retinopathy. Prog Retin Eye Res. 2016:51:156-86.

98. Suez J, Zmora N, Segal E, Elinav E. The pros, cons, and many unknowns of probiotics. Nat Med. 2019;25(5):716-29.

99. BordaloTonucci L, Dos Santos KM, De Luces Fortes Ferreira CL, Ribeiro SM, De Oliveira LL, Martino HS. Gut microbiota and probiotics: focus on diabetes mellitus. Crit Rev Food Sci Nutr. 2017;57(11):2296-309.

100. Razmpoosh E, Javadi A, Ejtahed HS, et al. The effect of probiotic supplementation on glycemic control and lipid profile in patients with type 2 diabetes: a randomized placebo controlled trial. Diabetes Metab Syndr. 2019;13(1):175-82.

101. Sato J, Kanazawa A, Azuma K, Ikeda F, et al. Probiotic reduces bacterial translocation in type 2 diabetes mellitus: A randomised controlled study. Sci Rep. 2017;7(1):12115. 
102. Bagarolli RA, Tobar N, Oliveira AG, Araújo TG, Carvalho BM, Rocha GZ, Vecina JF, Calisto K, Guadagnini D, Prada PO, SantosA SSTO, Saad MJ. Probiotics modulate gut microbiota and improve insulin sensitivity in DIO mice. J Nutr Biochem. 2017;50:16-25.

103. Yamazaki T, Suzuki H, Yamada S, et al. Lactobacillus paracasei KW3110 suppresses inflammatory stress-induced premature cellular senescence of human retinal pigment epithelium cells and reduces ocular disorders in healthy humans. Int J Mol Sci. 2020;21(14):5091.

104. Kumar P, Kizhakkedathu JN, Straus SK. Antimicrobial peptides: diversity, mechanism of action and strategies to improve the activity and biocompatibility in vivo. Biomolecules. 2018;8(1):4

105. Lehrer RI. Primate defensins. Nat Rev Microbiol. 2004;2(9):727-38.

106. Pfalzgraff A, Brandenburg K, Weindl G. Antimicrobial peptides and their therapeutic potential for bacterial skin infections and wounds. Front Pharmacol. 2018;9:1-23.

107. Hou J, Liu HY, Diao H, Yu H. The truncated human beta-defensin 118 can modulate lipopolysaccharide mediated inflammatory response in RAW264.7 macrophages. Peptides. 2021;136:170438.

108. Heinbockel L, Weindl G, Correa W, et al. Anti-Infective and Anti-Inflammatory Mode of Action of Peptide 19-2.5. Int J Mol Sci. 2021;22(3):1465.

109. Martin L, Horst K, Chiazza F, et al. The synthetic antimicrobial peptide 19-2.5 attenuates septic cardiomyopathy and prevents down-regulation of SERCA2 in polymicrobial sepsis. Sci Rep. 2016;6:37277.

110. Wu M, Pan H, Leng W, et al. Distribution of microbes and drug susceptibility in patients with diabetic foot infections in Southwest China. J Diabetes Res. 2018;2018:9817308.

111. Xie J, Li Y, Dai J, et al. Olfactory ensheathing cells grafted into the retina of RCS Rats Suppress Inflammation by Down-Regulating the JAK/STAT Pathway. Front Cell Neurosci. 2019;13:341.

112. Jha KA, Pentecost M, Lenin R, et al. TSG-6 in conditioned media from adipose mesenchymal stem cells protects against visual deficits in mild traumatic brain injury model through neurovascular modulation. Stem Cell Res Ther. 2019;10(1):318.

113. Xian P, Hei Y, Wang R, Wang T, Yang J, Li J, Di Z, Liu Z, Baskys A, Liu W, Wu $\mathrm{S}$, Long Q. Mesenchymal stem cell-derived exosomes as a nanotherapeutic agent for amelioration of inflammation-induced astrocyte alterations in mice. Theranostics. 2019;9(20):5956-75.

114. Wahid F, Shehzad A, Khan T, Kim YY. MicroRNAs: synthesis, mechanism, function, and recent clinical trials. Biochim Biophys Acta. 2010;1803(11):1231-43.

115. Platania CBM, Maisto R, Trotta MC, et al. Retinal and circulating miRNA expression patterns in diabetic retinopathy: An in silico and in vivo approach. Br J Pharmacol. 2019;176(13):2179-94.

116. Morris DR, Bounds SE, Liu H, et al. Exosomal MiRNA transfer between retinal microglia and RPE. Int J Mol Sci. 2020;21(10):3541.

117. Fernando $\mathrm{N}$, Wong $\mathrm{JHC}$, Das S, et al. MicroRNA-223 regulates retinal function and inflammation in the healthy and degenerating retina. Front Cell Dev Biol. 2020;8:516.

118. Dong N, Wang Y. MiR-30a Regulates S100A12-induced retinal microglial activation and inflammation by targeting NLRP3. Curr Eye Res. 2019;44(11):1236-43.

119. Karali M, Guadagnino I, Marrocco E, et al. AAV-miR-204 protects from retinal degeneration by attenuation of microglia activation and photoreceptor cell death. Mol Ther Nucleic Acids. 2020;19:144-56.

120. Wang $Y$, Chen $S$, Wang J, et al. MicroRNA-93/STAT3 signalling pathway mediates retinal microglial activation and protects retinal ganglion cells in an acute ocular hypertension model. Cell Death Dis. 2021;12(1):41.

121. Burgaletto C, Platania CBM, Di Benedetto G, et al. Targeting the miRNA155 /TNFSF10 network restrains inflammatory response in the retina in a mouse model of Alzheimer's disease. Cell Death Dis. 2021;12(10):905.

122. Yan L, Lee S, Lazzaro DR, Aranda J, Grant MB, Chaqour B. Single and compound knock-outs of MicroRNA (miRNA)-155 and its angiogenic gene target CCN1 in mice alter vascular and neovascular growth in the retina via resident microglia. J Biol Chem. 2015;290(38):23264-81.

123. Schulte LN, Westermann AJ, Vogel J, et al. Differential activation and functional specialization of miR-146 and miR-155 in innate immune sensing. Nucleic Acids Res. 2013;41(1):542-53.

124. Amadio M, Pascale A, Cupri S, et al. Nanosystems based on siRNA silencing HuR expression counteract diabetic retinopathy in rat. Pharmacol Res. 2016;111:713-20.

\section{Publisher's Note}

Springer Nature remains neutral with regard to jurisdictional claims in published maps and institutional affiliations.
Ready to submit your research? Choose BMC and benefit from:

- fast, convenient online submission

- thorough peer review by experienced researchers in your field

- rapid publication on acceptance

- support for research data, including large and complex data types

- gold Open Access which fosters wider collaboration and increased citations

- maximum visibility for your research: over $100 \mathrm{M}$ website views per year

At BMC, research is always in progress.

Learn more biomedcentral.com/submissions 